\title{
FOR ONE SYSTEM OF DIFFERENTIAL EQUATIONS TAKEN AS DUAL OF THE LORENZ SYSTEM
}

\author{
Boro M. Piperevski
}

\begin{abstract}
This paper looks at and goes through Lorenz system and his dual system of differential equations. Within analytics we have come to some commonalities in both systems.
\end{abstract}

\section{INTRODUCTION}

Here we have the Lorenz system:

$$
\begin{aligned}
& \dot{x}=\sigma(y-x), \\
& \dot{y}=x(r-z)-y, \\
& \dot{z}=x y-b z, \\
& \sigma, r, b>0 .
\end{aligned}
$$

In literature $[1,7,8]$ it is known that system (1) has three singularities (fixed point, equilibrium point) in the points

$$
O(0,0,0), O_{1}(\sqrt{b(r-1)}, \sqrt{b(r-1)}, r-1), O_{2}(-\sqrt{b(r-1)},-\sqrt{b(r-1)}, r-1)
$$

for $r>1$, and one singularity in point $O(0,0,0)$, for $0<r<1$.

In accordance with the theory of stability $[1,2,3,5,7,8]$, due to importance system (1) stability examination for singularities we calculate appropriate characteristic equations.

The characteristic equation, for system (1), is given with

$$
(\lambda+b)\left[\lambda^{2}+(\sigma+1) \lambda+\sigma(1-r)\right]=0,
$$

2010 Mathematics Subject Classification. Primary: 37-G35, 34-C23, Secondary 34-K18 Key words and phrases. system of differential equations, dual system of Lorenz system, singularities (fixed point, equilibrium point), characteristic equations 
for $r>0$ in singularity $O(0,0,0)$.

The characteristic equation is given with

$$
\lambda^{3}+(\sigma+b+1) \lambda^{2}+b(\sigma+r) \lambda+2 \sigma b(r-1)=0,
$$

for $r>1$ in singularities

$$
O_{1}(\sqrt{b(r-1)}, \sqrt{b(r-1)}, r-1), \quad O_{2}(-\sqrt{b(r-1)},-\sqrt{b(r-1)}, r-1) .
$$

Lemma 1. Let an algebraic equation of third degree is given

$$
x^{3}+a_{1} x^{2}+a_{2} x+a_{3}=0, a_{1}, a_{2}, a_{3} \in \mathbf{R} .
$$

This equation has two conjugated pure imaginary roots if following condition is met [1]

$$
a_{1} a_{2}=a_{3}
$$

\section{MAIN RESUlt}

Let us bring transformation shift defined with

$$
x=x_{1}+i x_{2}, y=y_{1}+i y_{2}, z=z_{1}+i z_{2}
$$

where $x_{1}, x_{2}, y_{1}, y_{2}, z_{1}, z_{2}$ are real functions from real argument $t, i^{2}=-1$.

With direct substitute (5) in (1) we get system of differential equations

$$
\begin{aligned}
& \dot{x_{1}}=\sigma\left(y_{1}-x_{1}\right) \\
& \dot{y_{1}}=r x_{1}-\left(x_{1} z_{1}-x_{2} z_{2}\right)-y_{1} \\
& \dot{z_{1}}=x_{1} y_{1}-x_{2} y_{2}-b z_{1} \\
& \dot{x_{2}}=\sigma\left(y_{2}-x_{2}\right) \\
& \dot{y_{2}}=r x_{2}-\left(x_{1} z_{2}+x_{2} z_{1}\right)-y_{2} \\
& \dot{z_{2}}=x_{1} y_{2}+x_{2} y_{1}-b z_{2} .
\end{aligned}
$$

Lemma 2. The system of differential equations (6) has three singularities in

$$
\begin{gathered}
O(0,0,0,0,0,0), O_{1}(0,0, r-1, \sqrt{b(1-r)}, \sqrt{b(1-r)}, 0), \\
O_{2}(0,0, r-1,-\sqrt{b(1-r)},-\sqrt{b(1-r)}, 0),
\end{gathered}
$$

for $0<r<1$, and three singularities 


$$
\begin{gathered}
\left.O(0,0,0,0,0,0), O_{1}^{*}(\sqrt{b(r-1)}, \sqrt{b(r-1)}), r-1,0,0,0\right), \\
O_{2}^{*}(-\sqrt{b(r-1)},-\sqrt{b(r-1)}, r-1,0,0,0)
\end{gathered}
$$

for $r>1$.

Proof. By solving the algebraic equations system

$$
\begin{aligned}
& \sigma\left(y_{1}-x_{1}\right)=0 \\
& r x_{1}-\left(x_{1} z_{1}-x_{2} z_{2}\right)-y_{1}=0 \\
& x_{1} y_{1}-x_{2} y_{2}-b z_{1}=0 \\
& \sigma\left(y_{2}-x_{2}\right)=0 \\
& r x_{2}-\left(x_{1} z_{2}+x_{2} z_{1}\right)-y_{2}=0 \\
& x_{1} y_{2}+x_{2} y_{1}-b z_{2}=0,
\end{aligned}
$$

we get the coordinates of singularities.

Lemma 3. For a system of differential equations (6) in common singularity $O(0,0,0,0,0,0)$ appropriate characteristic equation is given with

$$
(\lambda+b)^{2}\left[\lambda^{2}+(\sigma+1) \lambda+\sigma(1-r)\right]^{2}=0,
$$

for $r>0$, and in remaining singularities

$O_{1}(0,0, r-1, \sqrt{b(1-r)}, \sqrt{b(1-r)}, 0), \quad O_{2}(0,0, r-1,-\sqrt{b(1-r)},-\sqrt{b(1-r)}, 0)$,

$\left.O_{1}^{*}(\sqrt{b(r-1)}, \sqrt{b(r-1)}), r-1,0,0,0\right), \quad O_{2}^{*}(-\sqrt{b(r-1)},-\sqrt{b(r-1)}, r-1,0,0,0)$

appropriate characteristic equation is given with

$$
\left[\lambda^{3}+(\sigma+b+1) \lambda^{2}+b(\sigma+r) \lambda+2 \sigma b(r-1)\right]^{2}=0,
$$

in both cases $0<r<1$ and $r>1$.

Proof. Within calculating the matrix of Jacobi

$$
J(O)=\left[\begin{array}{cccccc}
-\sigma & \sigma & 0 & 0 & 0 & 0 \\
r & -1 & 0 & 0 & 0 & 0 \\
0 & 0 & -b & 0 & 0 & 0 \\
0 & 0 & 0 & -\sigma & \sigma & 0 \\
0 & 0 & 0 & r & -1 & 0 \\
0 & 0 & 0 & 0 & 0 & -b
\end{array}\right]
$$

and the corresponding determinant 


$$
\left|\begin{array}{cccccc}
-\sigma-\lambda & \sigma & 0 & 0 & 0 & 0 \\
r & -1-\lambda & 0 & 0 & 0 & 0 \\
0 & 0 & -b-\lambda & 0 & 0 & 0 \\
0 & 0 & 0 & -\sigma-\lambda & \sigma & 0 \\
0 & 0 & 0 & r & -1-\lambda & 0 \\
0 & 0 & 0 & 0 & 0 & -b-\lambda
\end{array}\right|,
$$

in common singularity $O(0,0,0,0,0,0)$, for $r>0$, we get characteristic equation (7).

With calculating the matrix of Jacobi $J\left(O_{1}^{*}\right)$

$$
\left[\begin{array}{cccccc}
-\sigma & \sigma & 0 & 0 & 0 & 0 \\
1 & -1 & -\sqrt{b(r-1)} & 0 & 0 & 0 \\
\sqrt{b(r-1)} & \sqrt{b(r-1)} & -b & 0 & 0 & 0 \\
0 & 0 & 0 & -\sigma & \sigma & 0 \\
0 & 0 & 0 & 1 & -1 & -\sqrt{b(r-1)} \\
0 & 0 & 0 & \sqrt{b(r-1)} & \sqrt{b(r-1)} & -b
\end{array}\right]
$$

and the corresponding determinant

$$
\left|\begin{array}{cccccc}
-\sigma- & \sigma & 0 & 0 & 0 & 0 \\
1 & -1- & -\sqrt{b(r-1)} & 0 & 0 & 0 \\
\sqrt{b(r-1)} & \sqrt{b(r-1)} & -b- & 0 & 0 & 0 \\
0 & 0 & 0 & -\sigma- & \sigma & 0 \\
0 & 0 & 0 & 1 & -1- & -\sqrt{b(r-1)} \\
0 & 0 & 0 & \sqrt{b(r-1)} & \sqrt{b(r-1)} & -b-
\end{array}\right|
$$

in singularity $\left.O_{1}^{*}(\sqrt{b(r-1)}, \sqrt{b(r-1)}), r-1,0,0,0\right)$, for $r>1$, we get characterristic equation (8).

With calculating the matrix of Jacobi $J\left(O_{1}\right)$ 


$$
\left[\begin{array}{cccccc}
-\sigma & \sigma & 0 & 0 & 0 & 0 \\
1 & -1 & 0 & 0 & 0 & \sqrt{b(r-1)} \\
0 & 0 & -b & -\sqrt{b(r-1)} & -\sqrt{b(r-1)} & 0 \\
0 & 0 & 0 & -\sigma & \sigma & 0 \\
0 & 0 & -\sqrt{b(r-1)} & 1 & -1 & 0 \\
\sqrt{b(r-1)} & \sqrt{b(r-1)} & 0 & 0 & 0 & -b
\end{array}\right]
$$

and the corresponding determinant

$$
\left|\begin{array}{cccccc}
-\sigma-\lambda & \sigma & 0 & 0 & 0 & 0 \\
1 & -1-\lambda & 0 & 0 & 0 & \sqrt{b(r-1)} \\
0 & 0 & -b-\lambda & -\sqrt{b(r-1)} & -\sqrt{b(r-1)} & 0 \\
0 & 0 & 0 & -\sigma-\lambda & \sigma & 0 \\
0 & 0 & -\sqrt{b(r-1)} & 1 & -1-\lambda & 0 \\
\sqrt{b(r-1)} & \sqrt{b(r-1)} & 0 & 0 & 0 & -b-\lambda
\end{array}\right|
$$

in singularity

$$
O_{1}(0,0, r-1, \sqrt{b(1-r)}, \sqrt{b(1-r)}, 0),
$$

for $0<r<1$, we get characteristic equation (8). We get the same equation (8) in other singularities

$$
O_{2}^{*}(-\sqrt{b(r-1)},-\sqrt{b(r-1)}, r-1,0,0,0),
$$

for $r>1$, and

$$
O_{2}(0,0, r-1,-\sqrt{b(1-r)},-\sqrt{b(1-r)}, 0)
$$

for $0<r<1$.

Note 1. Let us note that the characteristic equations (7) and (8) are in fact squares of the characteristic equations (2) and (3) for the Lorenz system. 


\section{DUAL SYSTEM OF THE LORENZ SYSTEM}

Let us now look at the system

$$
\begin{aligned}
& \dot{x_{2}}=\sigma\left(y_{2}-x_{2}\right) \\
& \dot{y_{2}}=r x_{2}-x_{2} z_{1}-y_{2} \\
& \dot{z_{1}}=-x_{2} y_{2}-b z_{1}
\end{aligned}
$$

gotten from the system (6) as his subsystem for $x_{1}=0, y_{1}=0, z_{2}=0$.

Definition. The system (9) is called a dual system of the Lorenz system.

Theorem 1. The system (9) has three singularities in the points

$$
O(0,0,0), O_{1}(\sqrt{b(1-r)}, \sqrt{b(1-r)}, r-1), O_{2}(-\sqrt{b(1-r)},-\sqrt{b(1-r)}, r-1),
$$

when limitation is $0<r<1$, and one singularity in point $O(0,0,0)$, when limitation is $r>1$.

Proof. From the algebraic equations system

$$
\begin{aligned}
& \sigma\left(y_{2}-x_{2}\right)=0, \\
& r x_{2}-x_{2} z_{1}-y_{2}=0, \\
& -x_{2} y_{2}-b z_{1}=0,
\end{aligned}
$$

we get singularities coordinates.

Theorem 2. For system (9), appropriate characteristic equation in singularity $O(0,0,0)$ is given with

$$
(\lambda+b)\left[\lambda^{2}+(\sigma+1) \lambda+\sigma(1-r)\right]=0,
$$

for $r>0$. For $0<r<1$ all roots of this equation (10) are negative. The characteristic equation in other singularities

$$
O_{1}(\sqrt{b(1-r)}, \sqrt{b(1-r)}, r-1), O_{2}(-\sqrt{b(1-r)},-\sqrt{b(1-r)}, r-1)
$$

is given with

$$
\lambda^{3}+(\sigma+b+1) \lambda^{2}+b(\sigma+r) \lambda+2 \sigma b(r-1)=0,
$$

for $0<r<1$. The characteristic equation (11) does not have purely imaginary roots.

Proof. The matrix of Jacobi of system (9) in singularity $O(0,0,0)$ is 


$$
J(O)=\left[\begin{array}{ccc}
-\sigma & \sigma & 0 \\
r & -1 & 0 \\
0 & 0 & -b
\end{array}\right]
$$

This means that the characteristic equation (10) is achieved with calculation of the determinant

$$
|J(O)-E|=\left|\begin{array}{ccc}
-\sigma-\lambda & \sigma & 0 \\
r & -1-\lambda & 0 \\
0 & 0 & -b-\lambda
\end{array}\right| .
$$

Equation of the roots (10) are

$$
\lambda_{1}=-b, \lambda_{2,3}=\frac{-(\sigma+1) \sqrt{(\sigma+1)^{2}-4 \sigma(1-r)}}{2}
$$

For $0<r<1$ we get

$$
-4 \sigma(1-r)<0,
$$

where from we have that all three roots are negative.

The Jacobi matrix of the system (9) in the singularity

$$
O_{1}(\sqrt{b(1-r)}, \sqrt{b(1-r)}, r-1),
$$

is

$$
J\left(O_{1}\right)=\left[\begin{array}{ccc}
-\sigma & \sigma & 0 \\
1 & -1 & -\sqrt{b(1-r)} \\
-\sqrt{b(1-r)} & -\sqrt{b(1-r)} & -b
\end{array}\right] .
$$

This means that the characteristic equation (11) is achieved with calculation of the determinant

$$
\left|J\left(O_{1}\right)-E\right|=\left|\begin{array}{ccc}
-\sigma-\lambda & \sigma & 0 \\
1 & -1-\lambda & -\sqrt{b(1-r)} \\
-\sqrt{b(1-r)} & -\sqrt{b(1-r)} & -b-\lambda
\end{array}\right| .
$$

We get the same equation (11) as well in the other singularity 


$$
O_{2}(-\sqrt{b(1-r)},-\sqrt{b(1-r)}, r-1) .
$$

According to the Lemma 1 and the condition (4), with application of the characteristic equation (11) we get the condition

$$
(1+\sigma+b) b(\sigma+r)=2 \sigma b(r-1)
$$

Due to the limitation $0<r<1$, the condition is not satisfied because the left side is always a positive number, and the right side is always a negative number.

Note 2. Let us note that characteristic equations (10) and (11) are the same with appropriate characteristic equations (2) and (3) for Lorenz system (1). Along with this it is important to point that for this characteristic equation the condition $0<r<1$ is valid, while for them at Lorenz system the condition $r>1$ is valid.

References

[1] J. E. Marsden, M. McCracken: The Hopf Bifurcation and its Applications, Springer-Verlag, New York 1976.

[2] G. Ioss, D. D. Joseph: Stability and Bifurcation Theory, Springer - Verlag, New York, Heidelberg, Berlin 1980.

[3] Arnold V. I.: Ordinary Differential Equations, MIT1978.

[4] E.L.Ince: Ordinary Differential Equations, Dover Publications INC. New York 1956.

[5] Л. С. Понтрягин: Обыкновенные дифференциальные уравнения, Наука, Москва1970.

[6] И. Г. Петровский: Лекции по теории обыкновенных дифференциальных уравнений, Наука, Москва, 1970.

[7] Schroeder M.: Fractals, Chaos, Power Laws, W.H.Freeman and Co., New York1991.

[8] R. Barrio, F. Blesa, S.Serrano: Behavior patterns in multiparametric

dynamical systems: Lorenz model, International Journal of Bifurcation and Chaos, Vol. 22, No. 6 (2012) 1230019

Faculty of Electrical Engineering and Information Technologies,

University "Ss Cyril and Methodius", Skopje, Macedonia,

E-mail address: borom@feit.ukim.edu.mk 